\title{
Understanding Angiography-Based Aneurysm Flow Fields through Comparison with Computational Fluid Dynamics
}

\author{
(D).R. Cebral, (D) F. Mut, (D).J. Chung, (D). Spelle, (D). Moret, (DF. van Nijnatten, and (DD. Ruijters
}

\begin{abstract}
BACKGROUND AND PURPOSE: Hemodynamics is thought to be an important factor for aneurysm progression and rupture. Our aim was to evaluate whether flow fields reconstructed from dynamic angiography data can be used to realistically represent the main flow structures in intracranial aneurysms.
\end{abstract}

MATERIALS AND METHODS: DSA-based flow reconstructions, obtained during interventional treatment, were compared qualitatively with flow fields obtained from patient-specific computational fluid dynamics models and quantitatively with projections of the computational fluid dynamics fields (by computing a directional similarity of the vector fields) in 15 cerebral aneurysms.

RESULTS: The average similarity between the DSA and the projected computational fluid dynamics flow fields was $78 \%$ in the parent artery, while it was only $30 \%$ in the aneurysm region. Qualitatively, both the DSA and projected computational fluid dynamics flow fields captured the location of the inflow jet, the main vortex structure, the intrasaccular flow split, and the main rotation direction in approximately $60 \%$ of the cases.

CONCLUSIONS: Several factors affect the reconstruction of 2D flow fields from dynamic angiography sequences. The most important factors are the 3-dimensionality of the intrasaccular flow patterns and inflow jets, the alignment of the main vortex structure with the line of sight, the overlapping of surrounding vessels, and possibly frame rate undersampling. Flow visualization with DSA from $>1$ projection is required for understanding of the $3 \mathrm{D}$ intrasaccular flow patterns. Although these DSA-based flow quantification techniques do not capture swirling or secondary flows in the parent artery, they still provide a good representation of the mean axial flow and the corresponding flow rate.

ABBREVIATIONS: CFD = computational fluid dynamics; MAFA = mean aneurysm flow amplitude (determined from DSA); MEAN = projection average; VEL = mean aneurysm velocity (determined from CFD)

V

isualization of in vivo aneurysmal flow structures and quantification of aneurysm hemodynamic characteristics is important in understanding the role of hemodynamics in the mechanisms responsible for wall degeneration and progression toward rupture or stabilization ${ }^{1}$ as well as for evaluating endovascular procedures such as flow diversion. ${ }^{2,3}$

Received October 18, 2016; accepted after revision January 25, 2017.

From the Bioengineering Department (J.R.C., F.M., B.J.C.), Volgenau School of Engineering, George Mason University, Fairfax, Virginia; Faculté de Médecine Paris-Sud (L.S.), Le Kremlin-Bicetre, France; Interventional Neuroradiology (J.M.), Beaujon University Hospital, Clichy, France; and Image Guided Therapy Innovation (F.v.N., D.R.) Philips Healthcare, Best, the Netherlands.

This work was supported by Philips Healthcare.

Please address correspondence to Juan R. Cebral, PhD, Bioengineering Department, Volgenau School of Engineering, George Mason University, 4400 University Dr, MSN 2A1, Fairfax, VA 22030; e-mail: jcebral@gmu.edu

Indicates article with supplemental on-line table.

Indicates article with supplemental on-line photo.

http://dx.doi.org/10.3174/ajnr.A5158
Previous studies have used computational fluid dynamics (CFD) to characterize the hemodynamic environment of the aneurysm to study aneurysm evolution ${ }^{4,5}$ and rupture. ${ }^{6-8}$ Other studies have used CFD to evaluate flow-diverting devices and procedures. ${ }^{9,10}$ On the other hand, imaging researchers have investigated using phase-contrast MR imaging to depict the in vivo flow fields within cerebral aneurysms, ${ }^{11}$ while others have developed flow-quantification methods from dynamic angiography. ${ }^{12,13} \mathrm{Vi}$ sualization and quantification of flow fields directly from angiography data are attractive because they can be performed directly in the angiography suite while imaging the aneurysm for diagnosis or treatment. Previous studies along this line have shown the potential clinical value of these techniques and have compared the results with those of Doppler sonography and synthetic angiography generated from CFD simulations. ${ }^{14}$

The purpose of our study was to analyze the flow fields reconstructed from dynamic angiography data by comparing them with patient-specific CFD models; in particular, we investigated 
whether these fields can be used to realistically represent the main intra-aneurysmal flow structures and identify limitations and factors that affect the flow field reconstruction.

\section{MATERIALS AND METHODS}

\section{Angiography-Based Flow Quantification}

Fifteen cerebral aneurysms with diameters of $>5 \mathrm{~mm}$, imaged with $3 \mathrm{D}$ rotational angiography and $2 \mathrm{D}$ digital subtraction angiography at 60 frames per second and a typical in-plane resolution of $0.29 \mathrm{~mm}$, were studied. Because the dose per frame is relatively low, the dose-area product level is comparable with a standard 3-frames per second DSA acquisition (dose-area product $=716 \mathrm{mGy} \cdot \mathrm{cm}^{2} / \mathrm{s}$ for the 60 -frames per second protocol versus $786 \mathrm{mGy} \cdot \mathrm{cm}^{2} / \mathrm{s}$ for the 3 -frames per second protocol). We acquired the 2D DSA sequences from 2 different viewpoints, trying to minimize the overlap between the aneurysm and the surrounding vessels. These sequences spanned approximately 7-12 cardiac cycles. In 2 patients, DSA sequences were acquired from a single projection, making a total of 28 sequences for all 15 patients.

2D flow fields in the aneurysms and surrounding vessels were reconstructed from the DSA sequences by using a previously developed technique based on an optical flow approach. ${ }^{12}$ Visualizations of these DSA flow fields were created by using virtual particle tracing (ie, a visualization technique based on integration of the equation of motion of massless particles to visualize velocity vector fields). Measurements of the instantaneous flow rate in the parent artery were obtained by integration of the velocity profile in ROIs placed on the proximal parent artery. The mean aneurysm flow amplitude (MAFA) was calculated by averaging the velocity magnitude over an ROI delineating the aneurysm contour. ${ }^{13}$

\section{Computational Fluid Dynamics Modeling}

Computational fluid dynamics models with patient-specific geometries were constructed from the $3 \mathrm{D}$ rotational angiography images by using previously described methods. ${ }^{15}$ We performed pulsatile flow simulations by numerically solving the 3D incompressible Navier-Stokes equations, assuming rigid walls and Newtonian fluid. ${ }^{16}$ These assumptions seem reasonable because aneurysm walls in general do not undergo large displacements, and shear thinning effects do not have enough time to develop in aneurysm flows. ${ }^{16}$ The maximum element size was set to $0.02 \mathrm{~cm}$, and a minimum of 10 points across any vessel cross-section was specified. The resulting number of elements ranged from 3 to 4 million tetrahedra. The time-dependent flow rate measurements obtained in the parent artery from the DSA sequences were used to prescribe patient-specific inflow boundary conditions. The simulations were performed for all cardiac cycles covered by the dynamic DSA sequences, by using 120 time-steps per cycle. To avoid possible imprecisions due to the initialization of the flow calculations, we discarded data from the first cardiac cycle. The resulting CFD fields were saved at 60 frames per cycle, coinciding with the time instants of the DSA sequences. The mean aneurysm velocity (VEL) was calculated as the average of the $3 \mathrm{D}$ velocity magnitude over the aneurysm region and over the cardiac cycles and compared with the MAFA.
Similarity of DSA and MEAN CFD projected flow fields in the region of the vessel, aneurysm, and both regions combined

\begin{tabular}{lcccc}
\hline Patient & View & Vessel & Aneurysm & Combined \\
\hline 1 & 1 & $80.3 \%$ & $66.0 \%$ & $76.2 \%$ \\
2 & 1 & $93.9 \%$ & $52.7 \%$ & $76.7 \%$ \\
3 & 1 & $80.5 \%$ & $46.4 \%$ & $76.3 \%$ \\
& 2 & $91.3 \%$ & $39.4 \%$ & $86.0 \%$ \\
4 & 1 & $59.6 \%$ & $55.9 \%$ & $57.9 \%$ \\
& 2 & $73.4 \%$ & $50.2 \%$ & $67.9 \%$ \\
5 & 1 & $91.4 \%$ & $-23.0 \%$ & $48.0 \%$ \\
& 2 & $81.9 \%$ & $-64.6 \%$ & $20.3 \%$ \\
6 & 1 & $71.4 \%$ & $66.8 \%$ & $70.7 \%$ \\
& 2 & $71.3 \%$ & $41.8 \%$ & $66.8 \%$ \\
7 & 1 & $57.6 \%$ & $-1.0 \%$ & $46.8 \%$ \\
& 2 & $74.3 \%$ & $9.8 \%$ & $64.7 \%$ \\
8 & 1 & $78.9 \%$ & $12.5 \%$ & $71.2 \%$ \\
& 2 & $77.3 \%$ & $-2.0 \%$ & $66.9 \%$ \\
9 & 1 & $80.0 \%$ & $32.8 \%$ & $69.5 \%$ \\
& 2 & $85.2 \%$ & $65.0 \%$ & $79.6 \%$ \\
10 & 1 & $66.8 \%$ & $67.9 \%$ & $67.3 \%$ \\
& 2 & $83.0 \%$ & $84.9 \%$ & $83.7 \%$ \\
11 & 1 & $88.5 \%$ & $25.7 \%$ & $73.3 \%$ \\
& 2 & $81.5 \%$ & $11.5 \%$ & $68.7 \%$ \\
12 & 1 & $71.4 \%$ & $46.1 \%$ & $66.4 \%$ \\
& 2 & $82.9 \%$ & $44.2 \%$ & $76.8 \%$ \\
13 & 1 & $77.5 \%$ & $63.1 \%$ & $72.6 \%$ \\
& 2 & $93.6 \%$ & $9.8 \%$ & $72.5 \%$ \\
14 & 1 & $74.2 \%$ & $15.3 \%$ & $62.8 \%$ \\
& 2 & $58.3 \%$ & $25.5 \%$ & $52.1 \%$ \\
& 1 & $73.2 \%$ & $-0.6 \%$ & $43.2 \%$ \\
& 1 & $80.5 \%$ & $26.8 \%$ & $56.2 \%$ \\
\hline \multirow{3}{*}{15} & 2 & $78.4 \% \pm 10.1 \%$ & $30.4 \% \pm 32.0 \%$ & $66.0 \% \pm 13.7 \%$ \\
\hline
\end{tabular}

For comparison, the CFD flow fields were projected to the same views used for the DSA acquisitions. This projection results in a $2 \mathrm{D}$ vector field on the imaging plane normal to the line of sight. Because the $3 \mathrm{D}$ rotational angiography images used to reconstruct the CFD models and the 2D DSA sequences were acquired relative to the same reference frame, this projection was straightforward (ie, it did not require any image coregistration). During the projection, the CFD velocity components along the line of sight were discarded. The remaining in-plane components were averaged along the line of sight. All CFD mesh points mapped to the same DSA pixels were averaged (for a MEAN or average projection), or the vector with the maximum magnitude was taken (for an MIP projection). The projected 2D CFD flow fields were visualized in a manner similar to the DSA fields by using virtual particle tracing.

\section{Data Analysis}

The 2D DSA and CFD flow fields were quantitatively compared by using a directional similarity measure $s$ defined as

$$
s=\frac{1}{N} \sum_{i \in R O I} \frac{v_{\mathrm{i}} \cdot u_{\mathrm{i}}}{\left|v_{\mathrm{i}}\right|\left|u_{\mathrm{i}}\right|}=100,
$$

where $v_{\mathrm{i}}$ is the DSA velocity vector; $u_{\mathrm{i}}$, the projected CFD velocity vector; $R O I$, the region of interest (aneurysm or parent vessel); $N$, the number of pixels in the ROI; and the dot operator denotes the dot product. This quantity measures the similarity of the directions of the 2 vector fields over the ROI. A similarity of $100 \%$ means a perfect match, random input would yield a $0 \%$, and op- 
VEL vs MAFA

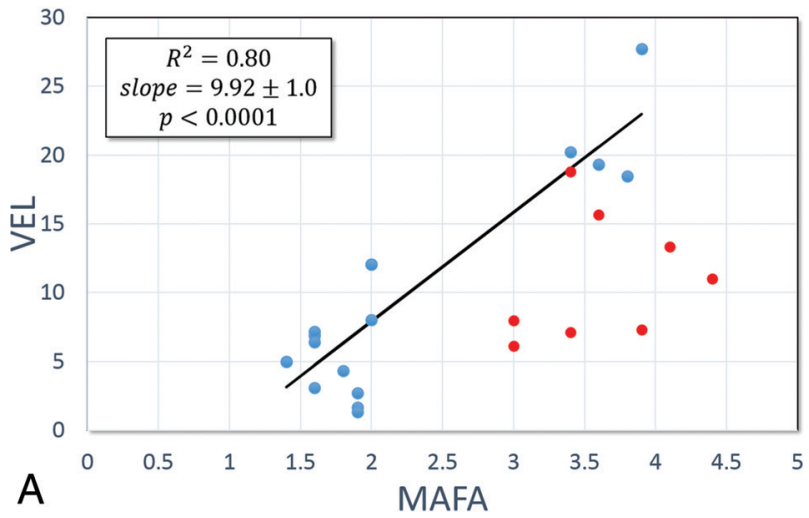

MAFA/VEL vs \#Frames

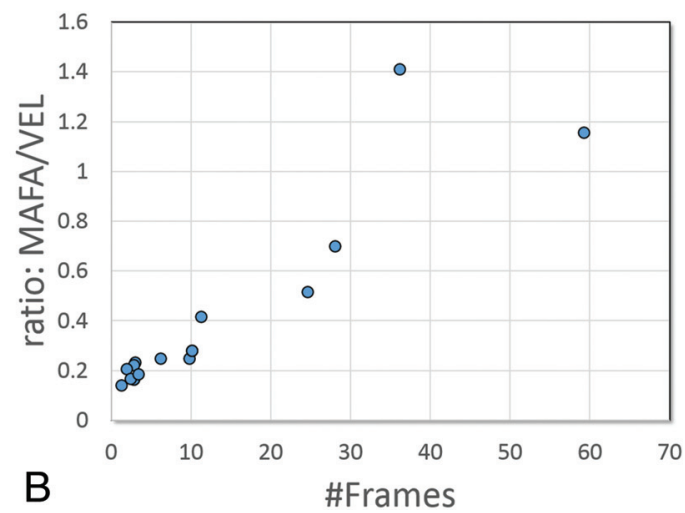

FIG 1. A, Linear correlation between the MAFA and VEL. Red dots represent cases discarded from the regression analysis due to substantial overlap between the aneurysm and surrounding vessels in the selected DSA view. $B$, Ratio of MAFA/VEL as a function of the number of frames needed for a particle to traverse the aneurysm diameter (mean aneurysm transit time).
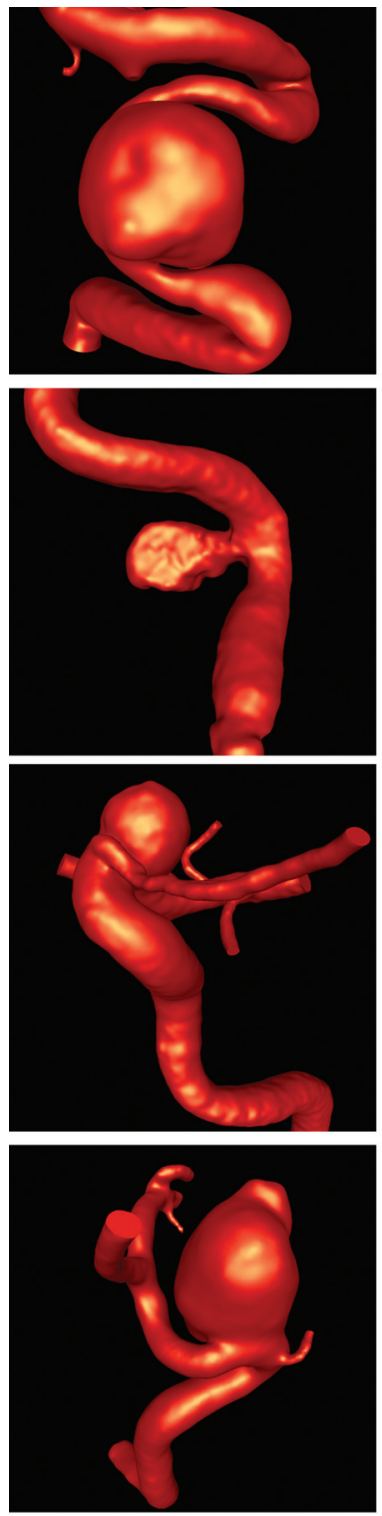
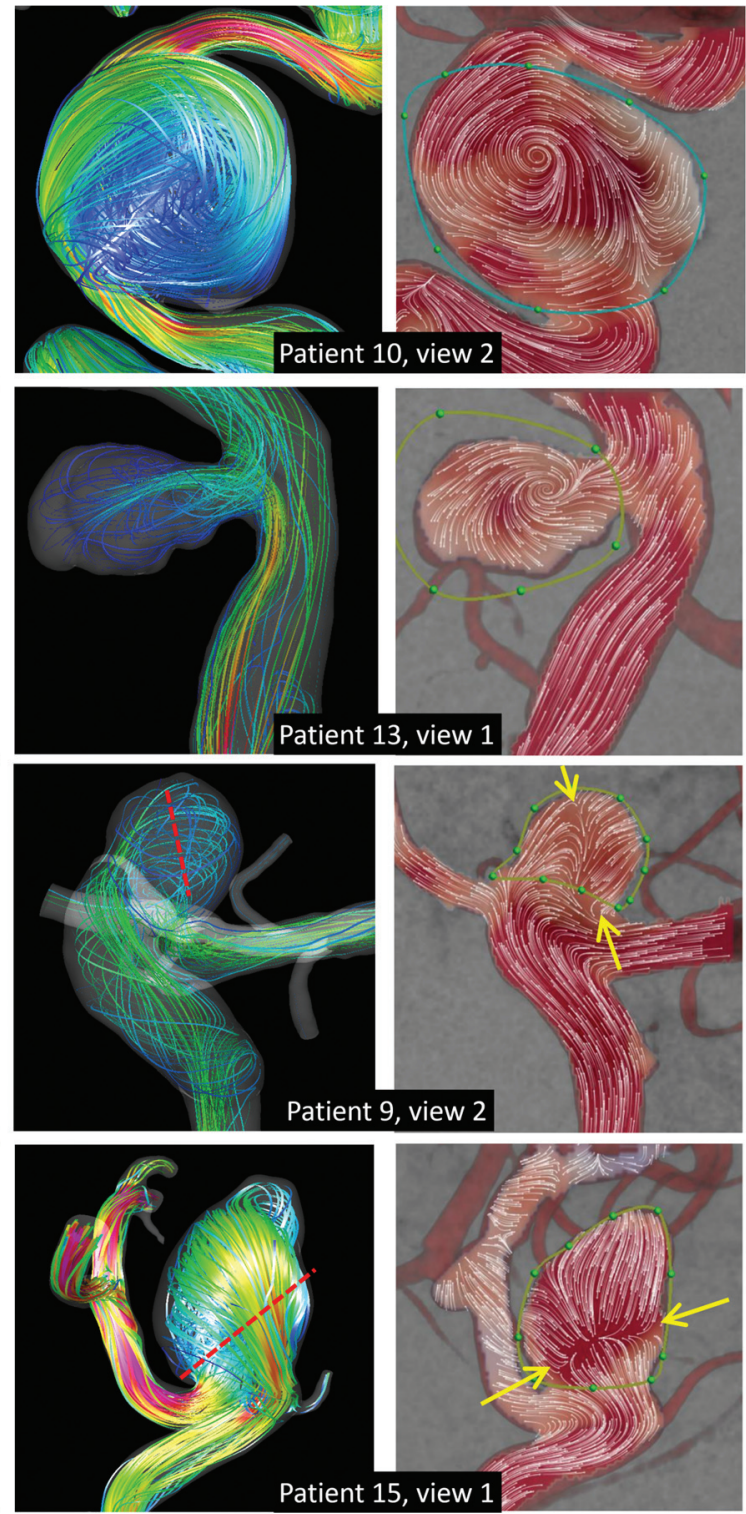
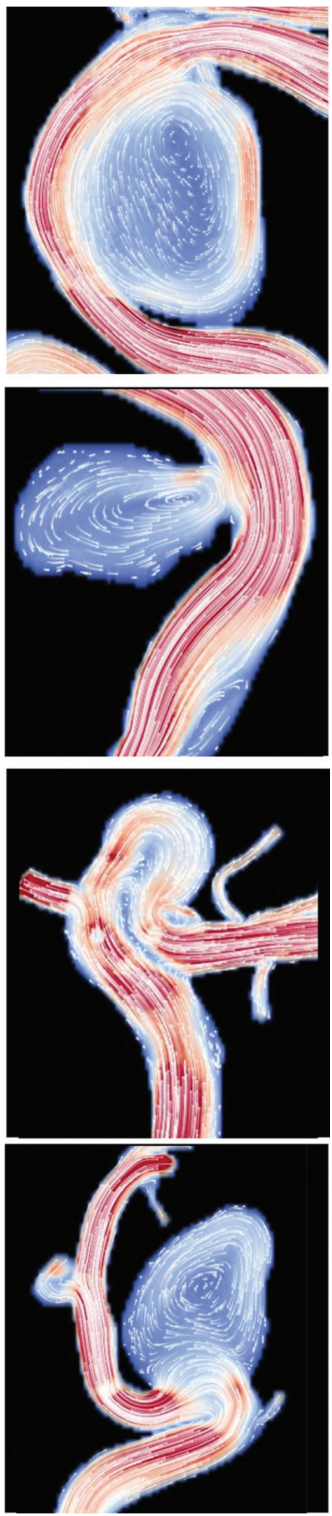

FIG 2. Examples of 4 aneurysms (rows) with vortex structures with varying alignment with the line of sight of DSA sequences. From left to right, columns show the following: reconstructed CFD model, visualization of 3D flow field by using streamlines, 2D DSA flow field, and 2D projected MEAN CFD flow field. Dotted red lines indicate the location of the vortex in the 3D flow. Yellow arrows indicate flow artifacts (divergence of particle paths) in the DSA flow reconstruction aligned with the vortex centers. 

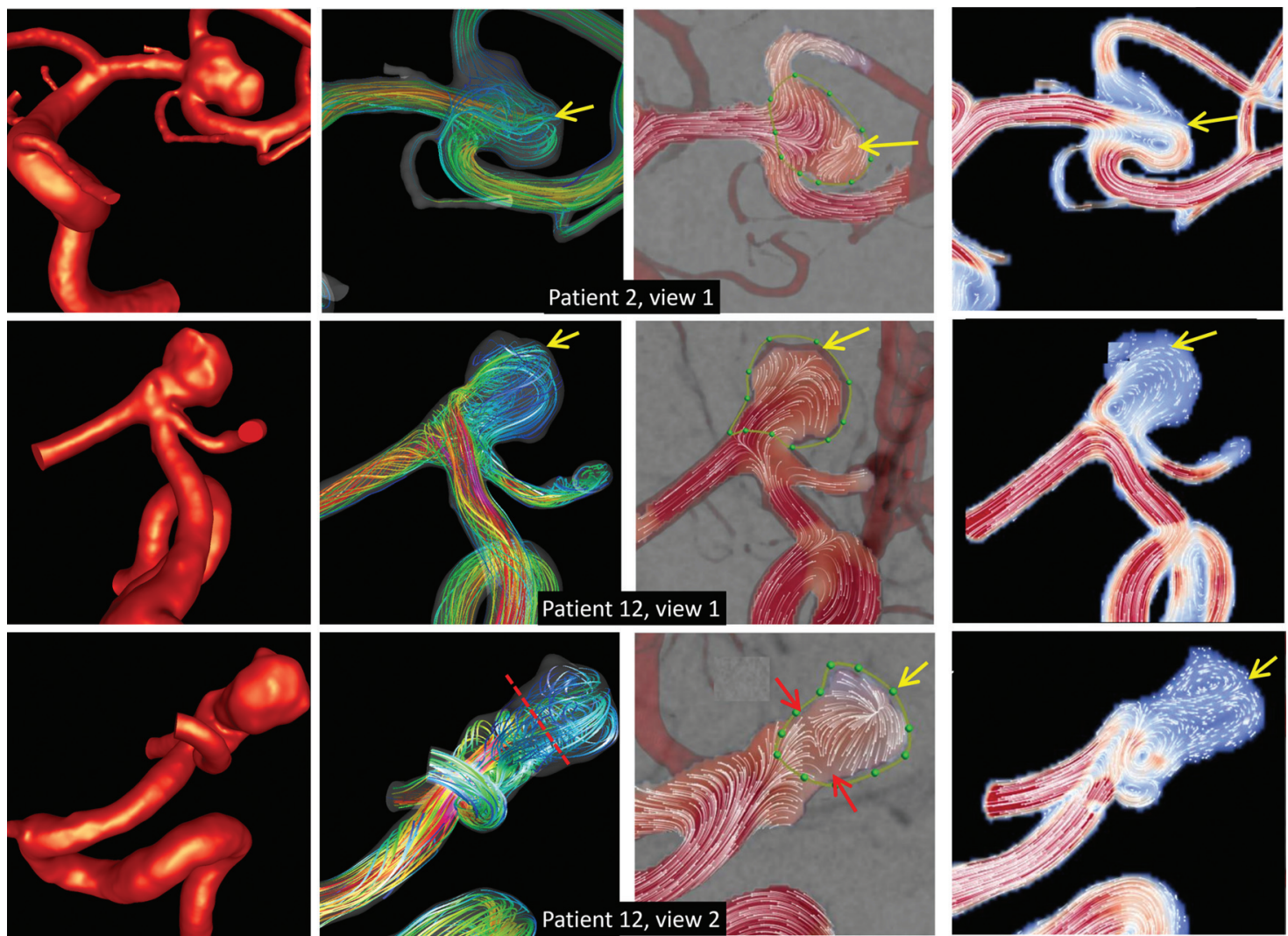

FIG 3. Top row: an example of when the DSA flow visualization does not depict the intrasaccular flow split. Center and bottom rows: an example of when the DSA flow visualizations from 2 roughly normal projections depict the intrasaccular flow split and allow understanding of the 3D flow structure. From left to right, columns show the reconstructed CFD model, visualization of 3D flow field by using streamlines, 2D DSA flow field, and the 2D projected MEAN CFD flow field. Yellow arrows point to the region of flow split. Red dotted line indicates center of rotation, and red arrows, the "convergent vectors" effect.

posing fields would give a $-100 \%$. Similarities of the 2 D DSA and CFD fields were calculated for the aneurysm and parent artery regions separately and for both regions combined.

The DSA flow fields and the 2D projected CFD fields were qualitatively compared with visualizations of the $3 \mathrm{D}$ flow fields obtained from the CFD models by using streamlines. These comparisons were performed to evaluate whether the DSA or the projected CFD fields were able to depict the location of the inflow jet, the main vortex structure within the aneurysm, the flow split within the aneurysm (if any), the direction of flow rotation within the aneurysm, and the swirling or secondary flows in the parent artery.

\section{RESULTS}

The directional similarity measures between the DSA and the projected CFD flow fields are presented in the Table for the aneurysm and vessel regions and for both regions combined. In the parent artery, the DSA and projected CFD flow fields are in good agreement with an average similarity of $78 \%$. In contrast, the average agreement within the aneurysm region alone is quite poor with a mean similarity of only $30 \%$.

To understand this discrepancy in the agreement of the DSA and CFD fields between the aneurysm and parent artery regions, we visually compared the $2 \mathrm{D}$ fields with visualizations of the $3 \mathrm{D}$ field. The results are presented in the On-line Table. This table indicates whether the DSA or projected CFD fields capture differ- ent flow characteristics observed in the streamline visualizations of the 3D fields. As explained previously, the in-plane components of the projected CFD velocity were averaged along the line of sight. We denoted this field as MEAN. A second field was computed by keeping the in-plane vector with the largest magnitude, similar to a maximum intensity projection used for visualization of 3D images. We denoted this second field as MIP. The MIP field was introduced to highlight the effects of vessel overlaps and to better understand the effects of projection of $3 \mathrm{D}$ vector fields onto a 2D plane. The On-line Table includes results for both the MEAN and MIP fields. The results indicate that the DSA and MEAN CFD flow fields often fail to capture many of the flow features of interest (ie, they only provide reasonable representations in $<60 \%$ of the cases). Furthermore, in many cases, certain features are captured by the DSA field but not by the MEAN CFD field or vice versa. Qualitatively, the MIP CFD fields give a better depiction of the intrasaccular flow structure and provide a direct visualization of vessel overlaps but cannot be used directly to quantify the similarity with the DSA fields because the MIP projection loses any depth information and vessel overlaps tend to distort the aneurysm fields as discussed below.

Linear regression analysis (Fig $1 A$ ) indicates that the mean aneurysm flow amplitude determined from 2D DSA is linearly correlated to the mean aneurysm velocity estimated from the CFD models after discarding views with noticeable overlaps of the an- 

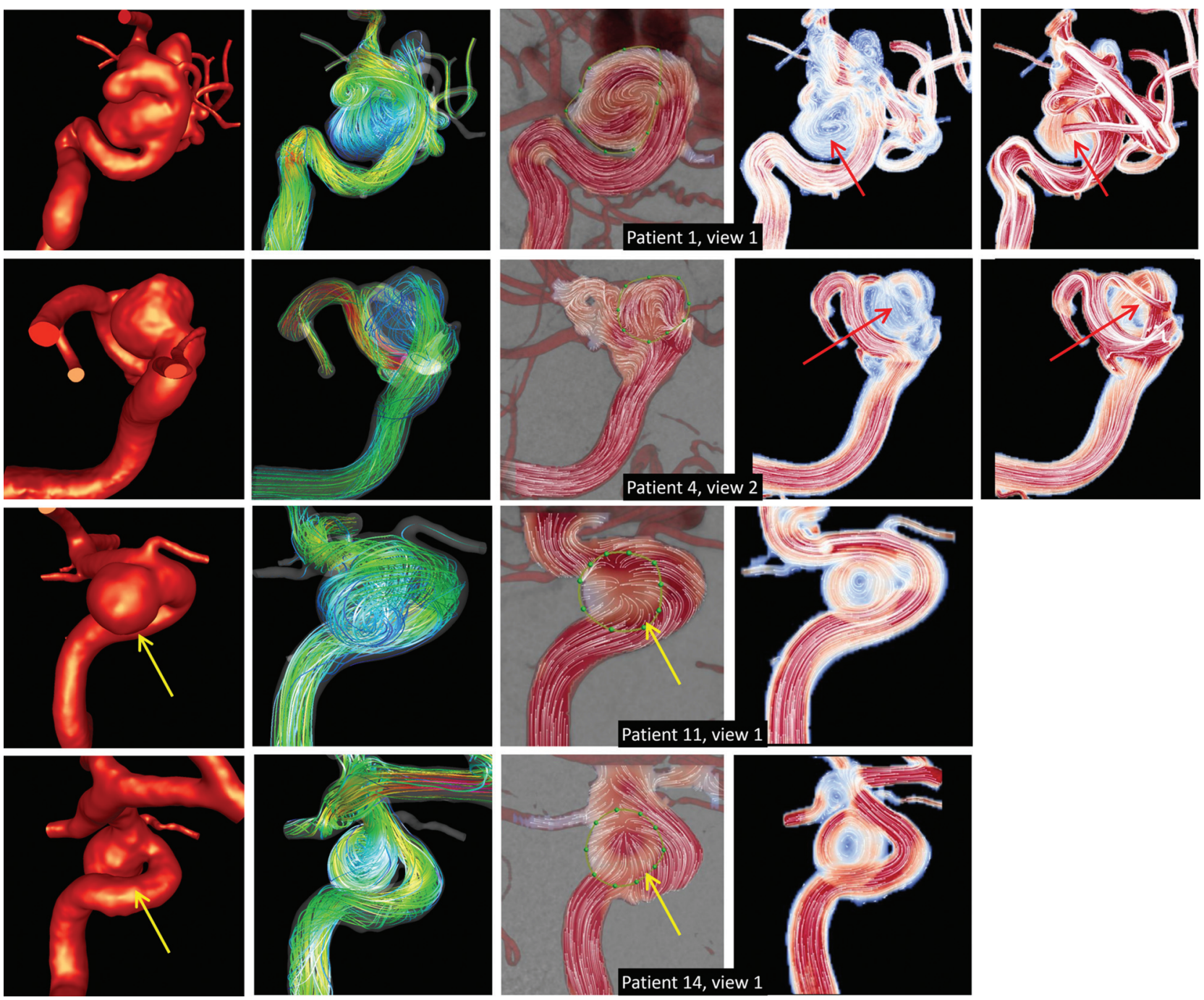

FIG 4. Examples of the effects of vessel overlaps on 4 aneurysms (rows). From left to right, columns show the following: the reconstructed CFD model, visualization of the 3D flow field by using streamlines, 2D DSA flow field, 2D projected MEAN CFD flow field, and 2D projected MIP CFD flow field. Red arrows show false vortex structures in projected CFD fields, while yellow arrows indicate false aneurysm inflow regions.

eurysm and surrounding vessels (slope $=7.92 \pm 1.00, R^{2}=0.80$ $P<.001)$. Vessel overlap was determined by inspection of the DSA and the projected CFD model and flow fields. Eight of the 25 DSA views were discarded (32\%). This correlation is in agreement with earlier work comparing the MAFA ratios generated by DSA and CFD simulations. ${ }^{17}$ This suggests that the MAFA is a good surrogate measure for VEL but needs to be interpreted carefully because it provides an underestimation of the aneurysm mean velocity because it discards velocity components along the line of sight.

\section{DISCUSSION}

Several factors can affect the flow field quantification from DSA data and the projection of 3D CFD flow fields. CFD is not a criterion standard for representing intra-aneurysmal flow fields; however, the comparison of DSA and CFD fields allows us to understand and interpret the flow structures observed in vivo with the DSA-based technique and to identify artifacts and limitations.

First, the alignment of the main intrasaccular vortex structure relative to the line of sight of the DSA projections can have an important effect on the reconstructed flow fields and the CFD projections. Four examples are presented in Fig 2 to illustrate this effect. In the first 2 examples (top two rows), the vortex core is roughly aligned with the line of sight and both the DSA and projected CFD field can depict the main vortex structure. In contrast, in the third and fourth examples (bottom 2 rows), the vortex core is roughly perpendicular to the line of sight. In these cases, the DSA flow field shows interesting artifacts along a line roughly aligned with the vortex core. Along this line, the flow fields seem to converge. To explain this effect, see the example on the bottom row. Below the vortex line, the traces point upward toward the line and are aligned with the inflow velocity near the anterior wall of the aneurysm. However, above this line, the traces point downward toward the line and are aligned with the velocity of the recirculating blood near the posterior wall of the aneurysm. Thus, this feature gives the impression of converging flow toward the vortex core line. The projected CFD fields provide a misleading representation of the flow field because in these cases, they give the impression that there is a vortex roughly aligned with the line 

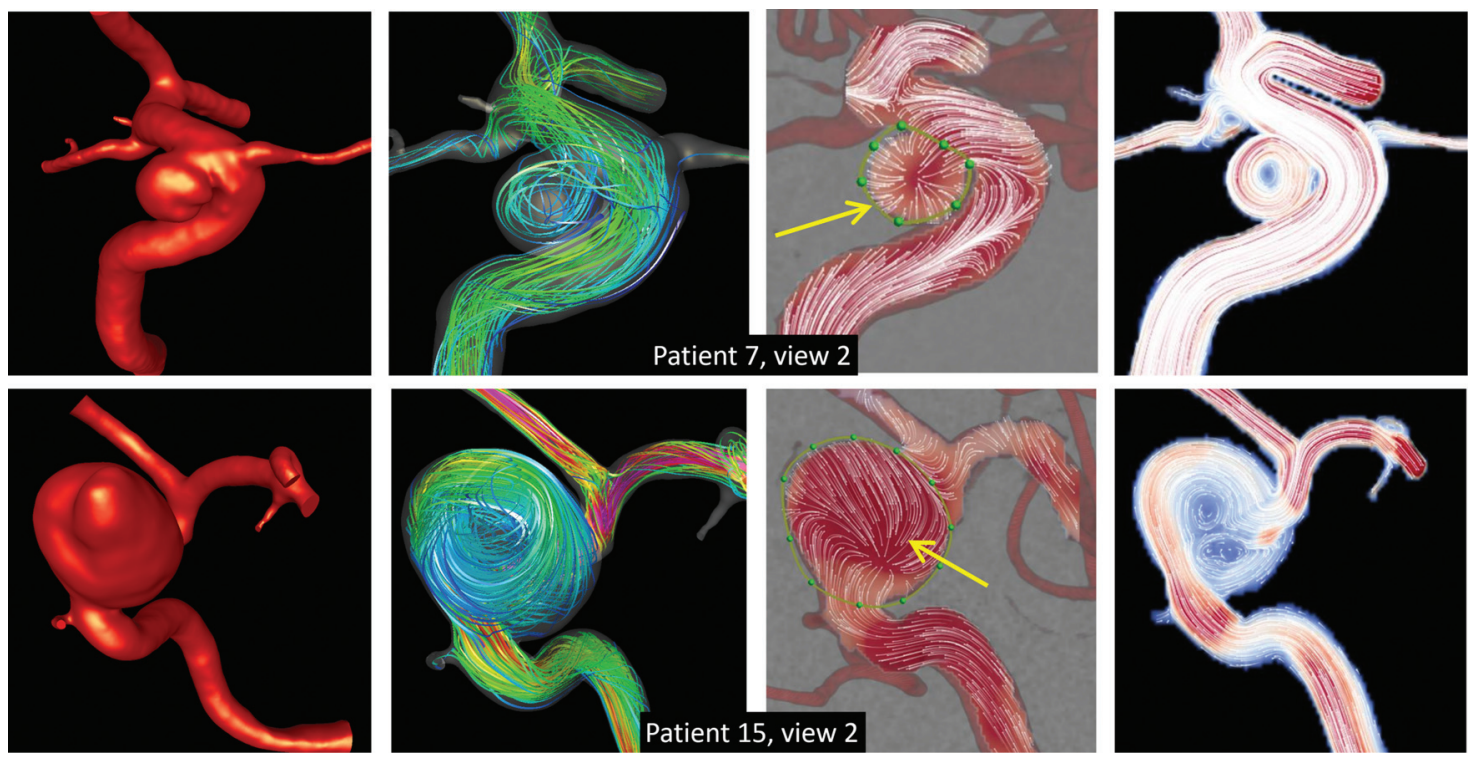

FIG 5. Examples of undersampling DSA flow fields in 2 aneurysms (rows). From left to right, columns show the following: the reconstructed CFD model, visualization of the 3D flow field by using streamlines, 2D DSA flow field, and 2D projected MEAN CFD flow field. Arrows point to the regions where fluid particles are observed to move across streamlines.

of sight when in reality, it is perpendicular to it. See the On-line Figure for further details.

Second, in cases in which the flow splits within the aneurysm cavity, the correct representation of the flow split by the DSA and projected CFD flow fields depends on the location of the inflow stream in $3 \mathrm{D}$ as well as the $3 \mathrm{D}$ structure of the recirculation regions. Two examples are presented in Fig 3. In the first example (top row), the inflow stream is located near the posterior wall of the vessel and the flow recirculates toward the anterior wall before flowing into the daughter branches. In this case, the flow split is properly visualized by the MEAN CFD field but not by the DSA field. In the second case (center row), the inflow stream is located near the anterior wall of the aneurysm and both the DSA and projected CFD fields provide adequate visualizations of the flow split. Furthermore, it is important to visualize the flow from $>1$ projection to understand the $3 \mathrm{D}$ flow structures. The bottom row of Fig 3 shows a second projection of the second example of this figure. In this second projection, the flow split is still visible in the DSA field, as well as the effect of the converging vectors when the main vortex is perpendicular to the line of sight described previously. Taken together, the DSA flow visualizations from the 2 roughly normal projections (Fig 3, center and bottom rows) provide a picture that allows us to understand the main structures of the 3D flow field.

Third, overlapping of the aneurysm with surrounding vessels for a given view point can affect the projected MEAN CFD flow fields by, for instance, generating false vortex structures. Examples of these kinds of distortions are presented in Fig 4 and are indicated by the red arrows. The MIP CFD fields shown in this figure clearly illustrate the effect of overlapping vessels on the field averaged along the line of sight and also illustrate why the MIP fields are also not appropriate for evaluating the DSA fields. On the other hand, vessel overlaps can affect the reconstruction of flow fields from DSA sequences by, for instance, generating false inflow or outflow regions, as illustrated in Fig 4 and indicated by the yellow arrows. Thus, vessel overlaps can affect the DSA and projected CFD fields differently; these different results can lead to poor similarity between these fields.

Finally, in cases in which the displacements of fluid particles in 1 timeframe are comparable with the dimensions of the aneurysm, an interesting effect can be observed in which particle traces seem to jump across streamlines instead of following them. This undersampling effect is illustrated in Fig 5 . The arrows point to regions where this effect is thought to take place. Note that this affects the DSA flow reconstruction but not the CFD projections; therefore, it can lead to poor similarity between the DSA and projected CFD fields. Because this can also affect the MAFA quantification, the difference (ratio) between MAFA and VEL is plotted in Fig $1 B$ as a function of mean aneurysm transit time or the number of frames required for fluid particles to traverse the aneurysm, estimated as Frames $=60 \times$ Aneurysm Diameter $/$ Mean Aneurysm Velocity. The difference decreases (the ratio becomes closer to 1) as the number of frames increases (the flow within the aneurysm is better resolved in time).

Most interesting, both the DSA and projected MEAN CFD flow fields neglect swirling or secondary flows in the parent artery but provide reasonable representations of the mean axial flow profile (which explains why the similarities are good in the vessel region). In the first example of Fig 2 (top row), the flow in the proximal parent artery has strong secondary flows shown by the streamline visualization and the MIP CFD projection, but not by the DSA or MEAN CFD fields. Similarly, in the first example of Fig 3 (top row), a strong swirling can be observed proximal to the internal carotid artery bifurcation in the streamline visualization, but the DSA or MEAN CFD fields give the impression of a perfect laminar parallel flow in this region.

\section{CONCLUSIONS}

Linear regression analysis suggests that the mean aneurysm flow amplitude determined from DSA is linearly correlated to the 
mean aneurysm velocity determined from CFD after discarding views with substantial vessel overlap.

While a good correspondence between the arterial flow fields detected in DSA and CFD reconstructions has been observed (directional similarity of $78 \%$ on average), the similarity fluctuated considerably for the aneurysm flow fields. Several factors affect the reconstruction of $2 \mathrm{D}$ aneurysm flow fields from angiography sequences. The most important factors are the 3-dimensionality of the intrasaccular flow patterns and inflow jets; the alignment of the main vortex structure with the line of sight; the overlapping of surrounding vessels, which many times is unavoidable; and possible frame-rate undersampling. Flow visualization with DSA from $>1$ projection is required for understanding the 3D intrasaccular flow patterns.

Although these DSA-based flow quantification techniques do not capture swirling or secondary flows in the parent artery, they still provide a good representation of the mean axial flow and the corresponding flow rate. This information is valuable for prescribing patient-specific flow conditions in CFD models of cerebral aneurysms used to understand mechanisms of aneurysm evolution and rupture and to evaluate endovascular procedures and devices.

Disclosures: Juan R. Cebral-UNRELATED: Grants/Grants Pending: National Institutes of Health, Philips Healthcare.* Fernando Mut-UNRELATED: Grant: National Institutes of Health.* Laurent Spelle-UNRELATED: Consultancy: Medtronic, Stryker. Jacques Moret—UNRELATED: Consultancy: MicroVention, Stryker, Medtronic. Fred van Nijnatten-UNRELATED: Employment: Philips Healthcare. Daniel RuijtersUNRELATED: Employment: Philips Healthcare. *Money paid to the institution.

\section{REFERENCES}

1. Sforza D, Putman CM, Cebral JR. Hemodynamics of cerebral aneurysms. Annu Rev Fluid Mech 2009;41:91-107 Medline

2. Chong W, Zhang Y, Qian Y, et al. Computational hemodynamics analysis of intracranial aneurysms treated with flow diverters: correlation with clinical outcomes. AJNR Am J Neuroradiol 2014;35: 136-42 CrossRef Medline

3. Sadasivan C, Cesar L, Seong J, et al. Treatment of rabbit elastaseinduced aneurysm models by flow diverters: development of quantifiable indexes of device performance using digital subtraction angiography. IEEE Trans Med Imaging 2009;28:1117-25 CrossRef Medline

4. Watton PN, Raberger NB, Holzapfel GA, et al. Coupling the hemodynamic environment to the evolution of cerebral aneurysms: computational framework and numerical examples. J Biomech Eng 2009;131:101003 CrossRef Medline

5. Sforza DM, Kono K, Tateshima S, et al. Hemodynamics in growing and stable cerebral aneurysms. J Neurointerv Surg 2016;8:407-12 CrossRef Medline

6. Shojima M, Oshima M, Takagi K, et al. Magnitude and role of wall shear stress on cerebral aneurysm: computational fluid dynamic study of $\mathbf{2 0}$ middle cerebral artery aneurysms. Stroke 2004;35: 2500-05 CrossRef Medline

7. Xiang J, Natarajan SK, Tremmel M, et al. Hemodynamic-morphologic discriminants for intracranial aneurysm rupture. Stroke 2011; 42:144-52 CrossRef Medline

8. Cebral JR, Mut F, Weir J, et al. Association of hemodynamic characteristics and cerebral aneurysm rupture. AJNR Am J Neuroradiol 2011;32:264-70 CrossRef Medline

9. Mut F, Raschi M, Scrivano E, et al. Association between hemodynamic conditions and occlusion times after flow diversion in cerebral aneurysms. J Neurointerv Surg 2015;7:286-90 CrossRef Medline

10. Huang Q, Xu J, Cheng J, et al. Hemodynamic changes by flow diverters in rabbit aneurysm models: a computational fluid dynamic study based on micro-computed tomography reconstruction. Stroke 2013;44:1936-41 CrossRef Medline

11. Wetzel S, Meckel S, Frydrychowicz A, et al. In vivo assessment and visualization of intracranial arterial hemodynamics with flow-sensitized 4D MR imaging at 3T. AJNR Am J Neuroradiol 2007;28: 433-38 Medline

12. Bonnefous O, Pereira VM, Ouared R, et al. Quantification of arterial flow using digital subtraction angiography. Med Phys 2012;39: 6264-75 CrossRef Medline

13. Pereira VM, Bonnefous O, Ouared R, et al. A DSA-based method using contrast-motion estimation for the assessment of the intraaneurysmal flow changes induced by flow-diverter stents. AJNR Am J Neuroradiol 2013;34:805-15 CrossRef Medline

14. Brina $\mathrm{O}$, Ouared $\mathrm{R}$, Bonnefous $\mathrm{O}$, et al. Intra-aneurysmal flow patterns: illustrative comparison among digital subtraction angiography, optical flow, and computational fluid dynamics. AJNR Am J Neuroradiol 2014;35:2348-53 CrossRef Medline

15. Cebral JR, Castro MA, Appanaboyina S,, et al. Efficient pipeline for image-based patient-specific analysis of cerebral aneurysm hemodynamics: technique and sensitivity. IEEE Trans Med Imaging 2005;24:457-67 CrossRef Medline

16. Cebral JR, Duan X, Gade PS, et al. Regional mapping of hemodynamics and wall characteristics of intracranial aneurysms. Ann Biomed Eng 2016;44:3553-67 CrossRef Medline

17. Van Nijnatten F, Bonnefuus O, Morales HG. Mean aneurysm flow amplitude ratio comparison between DSA and CFD. In: Navab N, Hornegger J, Wells W, Frangi A, eds. Medical Image Computing and Computer-Assisted Intervention - MICCAI 2015. Lecture Notes in Computer Science, vol 9350. Switzerland: Springer; 2015:485-92 CrossRef 